LU-ITP 2002/022

\title{
Spectral representation and dispersion relations in field theory on noncommutative space
}

\author{
Yi Liao, Klaus Sibold \\ Institut für Theoretische Physik, Universität Leipzig, \\ Augustusplatz 10/11, D-04109 Leipzig, Germany
}

\begin{abstract}
We study the spectral representation and dispersion relations that follow from some basic assumptions and the reduced spacetime symmetries on noncommutative (NC) space. Kinematic variables involving the NC parameter appear naturally as parametric variables in this analysis. When subtractions are necessary to remove ultraviolet divergences, they are always made at the fixed values of these $\mathrm{NC}$ variables. This point is also illustrated by a perturbative analysis of self-energies. Our analysis of the reduced spacetime symmetries suggests a weaker microcausality requirement. Starting from it, we make a first attempt at dispersion relations for forward scattering. It turns out that the attempt is hampered by a new unphysical region specified by a given motion in the NC plane which does not seem to be surmountable using the usual tricks. Implications for a possible subtraction and renormalization scheme for NC field theory in which the ultraviolet-infrared (UV/IR) mixing is removed are also briefly commented on.
\end{abstract}

PACS: 02.40.Gh, 11.55.Fv, 11.10.-z

Keywords: noncommutative spacetime, spectral representation, dispersion relations, causality 


\section{Introduction}

Quantum field theory on noncommutative (NC) spacetime has some intriguing features that result from its nonlocal nature of interactions. The quantum corrections that are effectively regularized in the ultraviolet (UV) by the nonlocality become singular again for some kinematic configurations including the infrared (IR) one. This is the so-called UV/IR mixing problem [臬] that hampers the implementation of the usual renormalization procedure. There are additional problems when time does not commute with space. A direct application of Feynman rules for which the only NC effect is the appearance of NC phases [2] leads to violation of unitarity [3]. However, as has been shown recently, a proper treatment of perturbation theory in this case does not yield the assumed Feynman rules and unitarity can be maintained without any problem [四, 5]. Another issue with timespace $\mathrm{NC}$ is that it seems to lead to acausal effects in scattering [6]. Although there is no solution to this so far, there is a hope that this could be resolved by a proper redefinition of wavefunctions and states in the NC context [7].

The above interesting results are based on explicit perturbative analysis. In this

work we shall study the related issue concerning analytic properties of Green functions and scattering amplitudes by starting from some basic assumptions in NC field theory. In particular we want to know how the Källen-Lehmann spectral representation can be generalized to the NC case, and whether it is still possible to use microcausality to derive simple dispersion relations for scattering amplitudes. As we shall see, the drastic changes in locality and spacetime symmetries will make things not so obvious and present new obstacles especially in deriving a dispersion relation for scattering. Our discussion will also give a clue on how to implement the renormalization program in the presence of the $\mathrm{UV} / \mathrm{IR}$ mixing.

An essential difference of NC field theory from ordinary theory is that Lorentz invariance is generally lost. Since the latter is one of the basic assumptions in ordinary field theory and facilitates important arguments in deriving dispersion relations, we shall first discuss the new situation regarding spacetime symmetries due to the introduction of noncommutativity, $\left[\hat{x}_{\mu}, \hat{x}_{\nu}\right]=i \theta_{\mu \nu}$. Our results will be directly applicable to NC scalar field theory formulated in terms of the star product.

First of all, the translational invariance is preserved which guarantees the energymomentum conservation in all processes. We shall be only concerned in this work with 
space-space NC. In this case, without loss of generality, we can always choose NC to be restricted in the (12)-plane; i.e., $\theta_{12}=-\theta_{21}=\theta>0$, with others vanishing. Since $\hat{x}_{0,3}$ are not involved in NC, invariance in Lorentz boosts remains in the 3-direction. The NC relation $\left[\hat{x}_{1}, \hat{x}_{2}\right]=i \theta$ is also invariant under rotations around the axis in the 3 -direction. Suppose the NC vector $\vec{\theta}$ is in the normal direction to the NC plane, then the remaining symmetries are summarized as boosts along and rotations around $\vec{\theta}$. In momentum space, typical examples of invariants involving momenta $p, q$ and $\theta_{\mu \nu}$ include $p \cdot \tilde{q}, \tilde{p}^{2}$ and $\tilde{p} \cdot \tilde{q}$, where $\tilde{p}_{\mu}=\theta_{\mu \nu} p^{\nu}$ is the NC momentum orthogonal to $p$. Denoting the components of the spatial momentum $\vec{p}$ in the $\vec{\theta}$ direction and in the NC plane as $p_{\|}$and $\vec{p}_{\perp}$ respectively, we have, $p \cdot \tilde{q}=\vec{\theta} \cdot\left(\vec{p}_{\perp} \times \vec{q}_{\perp}\right), \tilde{p}^{2}=-\theta^{2} \vec{p}_{\perp}^{2}$, and $\tilde{p} \cdot \tilde{q}=-\theta^{2} \vec{p}_{\perp} \cdot \vec{q}_{\perp}$. These invariants are in addition to the ordinary ones $p^{2}$ and $p \cdot q$.

For completeness, we also mention briefly the other cases concerning $\theta_{\mu \nu}$. For $\theta_{i j}=0$, we may choose $\theta_{03}=-\theta_{30}=\theta$, with others vanishing. The remaining symmetries are precisely the same as in the above case. The mixed case of $\theta_{0 i} \neq 0$ and $\theta_{i j} \neq 0$ is generally complicated and there are usually no remaining symmetries separately for boosts and rotations except for one special case. Defining $e^{i}=\theta^{0 i}$ and $b^{i}=1 / 2 \epsilon^{i j k} \theta^{j k}$, the special case corresponds to $\vec{e} \| \vec{b}$ for which invariance remains in boosts along and rotations around the preferred direction.

The rest of the paper is organized as follows. We study in the next section the two-point function and its spectral representation. A dispersion relation is then written down using the analytic properties implied in the representation. As an example, we demonstrate explicitly in section 3 that the dispersion relation is satisfied for the self-energy at one loop in $\varphi^{3}$ perturbation theory. In section 4 we attempt to derive a dispersion relation for forward scattering amplitudes. To this end we propose a microcausality requirement which is weaker than the usual one but is in accord with our symmetry analysis in this section. Its viability is further exemplified by a perturbative calculation. We show how a new unphysical region arises that hampers any simple dispersion relations for scattering amplitudes. We summarize our results and discuss their implications in the last section.

\section{Spectral representation}

We assume that the usual assumption in ordinary field theory about physical spectrum and its completeness is still a reasonable starting point in space-space NC field theory. 
The main difference is that only part of relativistic invariance is preserved in NC theory. Our discussion follows the usual development in axiomatic field theory [8] and is a generalization of the Källen-Lehmann spectral representation to the NC space.

Let us consider the two-point function defined in terms of the Heisenberg scalar field $\varphi$ and vacuum state $|\Omega\rangle$,

$$
\begin{aligned}
D^{\prime}(x, y) & =\langle\Omega|\varphi(x) \varphi(y)| \Omega\rangle \\
& =D^{\prime}(x-y)
\end{aligned}
$$

where translational invariance has been used. Using the completeness relation of the physical spectrum, we have,

$$
\begin{aligned}
D^{\prime}(z) & =\sum_{\alpha} \int \frac{d^{3} \vec{p}}{(2 \pi)^{3} 2 E_{\vec{p}, \alpha}}|\langle\Omega|\varphi(0)| \vec{p}, \alpha\rangle|^{2} e^{-i p_{+} \cdot z} \\
& =\sum_{\alpha} \int \frac{d^{4} p}{(2 \pi)^{3}} \tau\left(p_{0}\right) \delta\left(p^{2}-m_{\alpha}^{2}\right)|\langle\Omega|\varphi(0)| \vec{p}, \alpha\rangle|^{2} e^{-i p \cdot z} .
\end{aligned}
$$

Here $z=x-y, p_{+}^{\mu}=\left(E_{\vec{p}, \alpha}, \vec{p}\right)$ and $E_{\vec{p}, \alpha}=\sqrt{\vec{p}^{2}+m_{\alpha}^{2}}$ with $\alpha$ representing all other quantum numbers specifying a state. $\tau$ is the step function; we have reserved $\theta$ for the $\mathrm{NC}$ parameter.

In ordinary theory the matrix element appearing in the above equation is a Lorentz invariant and depends on $p$ only through $p^{2}=m_{\alpha}^{2}$. In the NC case, the symmetry argument is less restrictive so that additional dependence on $p$ is permitted. According to our discussion in the previous section, this dependence can occur only in the form of $\tilde{p}^{2}=-\theta^{2} \vec{p}_{\perp}^{2}$. Inserting the $\delta$ function identity, we obtain

$$
\begin{aligned}
D^{\prime}(z) & =\int_{0}^{\infty} d m^{2} \sum_{\alpha} \int \frac{d^{4} p}{(2 \pi)^{3}} \tau\left(p_{0}\right) \delta\left(p^{2}-m^{2}\right) \delta\left(m^{2}-m_{\alpha}^{2}\right)|\langle\Omega|\varphi(0)| \vec{p}, \alpha\rangle|^{2} e^{-i p \cdot z} \\
& =\int d m^{2} \int \frac{d^{4} p}{(2 \pi)^{3}} \tau\left(p_{0}\right) \delta\left(p^{2}-m^{2}\right) e^{-i p \cdot z} \rho\left(m^{2}, \tilde{p}^{2}\right) \\
& =\int d m^{2} \rho\left(m^{2},(i \tilde{\partial})^{2}\right) \int \frac{d^{4} p}{(2 \pi)^{3}} \tau\left(p_{0}\right) \delta\left(p^{2}-m^{2}\right) e^{-i p \cdot z} \\
& =\int d m^{2} \rho\left(m^{2},(i \tilde{\partial})^{2}\right) D\left(z, m^{2}\right),
\end{aligned}
$$

where $(\tilde{\partial})^{2}=\theta_{\mu \beta} \theta_{\gamma}^{\mu} \partial_{z}^{\beta} \partial_{z}^{\gamma}=-\theta^{2}\left[\left(\partial_{z^{1}}\right)^{2}+\left(\partial_{z^{2}}\right)^{2}\right], \rho\left(m^{2}, \tilde{p}^{2}\right)$ is the spectral density function for a fixed $\tilde{p}^{2}$,

$$
\rho\left(m^{2}, \tilde{p}^{2}\right)=\sum_{\alpha} \delta\left(m^{2}-m_{\alpha}^{2}\right)|\langle\Omega|\varphi(0)| \vec{p}, \alpha\rangle|^{2}
$$

and $D$ is the usual function defined for free fields,

$$
D\left(z, m^{2}\right)=\int \frac{d^{4} p}{(2 \pi)^{3}} \tau\left(p_{0}\right) \delta\left(p^{2}-m^{2}\right) e^{-i p \cdot z} .
$$


The above representation has interesting physical implications. Let us first consider the following vacuum expectation value of the commutator,

$$
\begin{aligned}
i \Delta^{\prime}(z) & =\langle\Omega|[\varphi(x), \varphi(y)]| \Omega\rangle \\
& =\int d m^{2} \rho\left(m^{2},(i \tilde{\partial})^{2}\right) i \Delta\left(z, m^{2}\right),
\end{aligned}
$$

with $i \Delta\left(z, m^{2}\right)=D\left(z, m^{2}\right)-D\left(-z, m^{2}\right)$. It is well-known that the distribution $\Delta\left(z, m^{2}\right)$ is singular on the light-cone and vanishes for space-like $z$. The subtle point here is that $\rho\left(m^{2}, \tilde{p}^{2}\right)$ is generally not a polynomial in $\tilde{p}^{2}$ of finite order. The integrand in the above equation involves derivatives of infinite order and is thus highly nonlocal in spatial z. It is not clear whether it persists to vanish for space-like $z$ after this manipulation of derivatives. A more careful analysis will be given in section 4 . The result is that $\Delta^{\prime}(z)$ vanishes when $z$ is space-like in the commutative direction, i.e., $z_{0}^{2}<z_{\|}^{2}$.

The second quantity that we would like to explore is the complete Feynman propagator,

$$
\begin{aligned}
i D_{F}^{\prime}(z) & =\langle\Omega|T(\varphi(x) \varphi(y))| \Omega\rangle \\
& =\tau\left(z_{0}\right) D^{\prime}(z)+\tau\left(-z_{0}\right) D^{\prime}(-z) \\
& =\int_{0}^{\infty} d m^{2} \rho\left(m^{2},(i \tilde{\partial})^{2}\right)\left[\tau\left(z_{0}\right) D\left(z, m^{2}\right)+\tau\left(-z_{0}\right) D\left(-z, m^{2}\right)\right] \\
& =\int d m^{2} \rho\left(m^{2},(i \tilde{\partial})^{2}\right) i D_{F}\left(z, m^{2}\right)
\end{aligned}
$$

where $i D_{F}$ is the Feynman propagator for free fields,

$$
i D_{F}\left(z, m^{2}\right)=\int \frac{d^{4} p}{(2 \pi)^{4}} \frac{i}{p^{2}-m^{2}+i \epsilon} e^{-i p \cdot z} .
$$

We stress that the third equality is possible only for $\theta_{0 i}=0$; otherwise the derivative operation cannot commute with the step function. Transforming into momentum space yields,

$$
\begin{aligned}
i \hat{D}_{F}^{\prime}(k) & =\int d^{4} z e^{i k \cdot z} i D_{F}^{\prime}(z) \\
& =\int_{0}^{\infty} d m^{2} \rho\left(m^{2}, \tilde{k}^{2}\right) \int d^{4} z e^{i k \cdot z} i D_{F}\left(z, m^{2}\right) \\
& =\int d m^{2} \rho\left(m^{2}, \tilde{k}^{2}\right) i \hat{D}_{F}\left(k^{2}, m^{2}\right)
\end{aligned}
$$

where $i \hat{D}_{F}\left(k^{2}, m^{2}\right)=i\left(k^{2}-m^{2}+i \epsilon\right)^{-1}$ is the Feynman propagator for free fields in momentum space. In deriving the second equality above, we have employed integration by parts in $z$ and ignored spatial surface terms. This was done in the same spirit as we cope with the cyclicity of star products in the action, and is consistent with the energymomentum conservation which implies that total derivative terms are ignorable in the action. The above equation thus generalizes the Källen-Lehmann representation to the 
NC space,

$$
\hat{D}_{F}^{\prime}\left(k^{2}, \tilde{k}^{2}\right)=\int_{0}^{\infty} d m^{2} \frac{\rho\left(m^{2}, \tilde{k}^{2}\right)}{k^{2}-m^{2}+i \epsilon} .
$$

The NC momentum $\tilde{k}$ appears here as a parametric variable. Thus, the ordinary arguments leading from the above representation to a dispersion relation still apply; namely, for independent but fixed $\tilde{k}^{2}, \hat{D}_{F}^{\prime}\left(k^{2}, \tilde{k}^{2}\right)$ is an analytic function in the complex $k^{2}$ plane except for some simple poles and a branch cut in the positive axis due to the occurrence of isolated states and continum thresholds. We can therefore write down the following Hilbert transform,

$$
\hat{D}_{F}^{\prime}\left(k^{2}, \tilde{k}^{2}\right)=\frac{1}{\pi} \int_{-\infty}^{\infty} d k^{\prime 2} \frac{\operatorname{Im} \hat{D}_{F}^{\prime}\left(k^{\prime 2}, \tilde{k}^{2}\right)}{k^{\prime 2}-k^{2}-i \epsilon},
$$

which is the dispersion relation for the two-point Green function on NC space. The lower delimiter in the above equation can be effectively replaced by the squared mass of the lowest physical state. In most physical situations where the integrand does not converge fast enough as $k^{\prime 2} \rightarrow \infty$, subtractions are necessary to make the integral well-defined. We stress that these subtractions in $k^{2}$ must be made for the same fixed $\tilde{k}^{2}$.

\section{Dispersion relation for self-energy: a perturbative example}

To illustrate analytic properties of the two-point functions studied in the preceding section, we consider here an explicit one-loop example in perturbation theory. A similar analysis has been made previously for massless fields at the same level by a straightforward but complicated computation of loop integrals [9]. Our method will be simpler without actually evaluating loop integrals and more general in that it is applicable to massive fields as well.

The complete Feynman propagator $\hat{D}^{\prime}\left(k^{2}, \tilde{k}^{2}\right)$ is related to the $1 \mathrm{PI}$ self-energy $\Sigma\left(k^{2}, \tilde{k}^{2}\right)$ by,

$$
\hat{D}^{\prime}\left(k^{2}, \tilde{k}^{2}\right)=\left[k^{2}-m^{2}-\Sigma\left(k^{2}, \tilde{k}^{2}\right)+i \epsilon\right]^{-1}
$$

Note that, as the 1PI functions are defined by Legendre transform from the complete Green functions, this relation itself does not invoke for perturbation theory. $\Sigma$ has similar analytic properties as $\hat{D}^{\prime}$ except that the latter has additional simple poles. We therefore 
have, for sufficently converging $\Sigma$ at infinity,

$$
\Sigma\left(k^{2}, \tilde{k}^{2}\right)=\frac{1}{\pi} \int_{-\infty}^{\infty} d k^{\prime 2} \frac{\operatorname{Im} \Sigma\left(k^{2}, \tilde{k}^{2}\right)}{k^{\prime 2}-k^{2}-i \epsilon} .
$$

For $\Sigma$ going to a constant as $k^{2} \rightarrow \infty$, one subtraction is necessary for the same fixed $\tilde{k}^{2}$,

$$
\Sigma\left(k^{2}, \tilde{k}^{2}\right)-\Sigma\left(\mu^{2}, \tilde{k}^{2}\right)=\frac{k^{2}-\mu^{2}}{\pi} \int_{-\infty}^{\infty} d k^{\prime 2} \frac{\operatorname{Im} \Sigma\left(k^{\prime 2}, \tilde{k}^{2}\right)}{\left(k^{\prime 2}-k^{2}-i \epsilon\right)\left(k^{\prime 2}-\mu^{2}-i \epsilon\right)},
$$

where $\mu^{2}$ is an arbitrary subtraction point. Now we demonstrate that the above is satisfied at one loop in $\varphi^{3}$ theory.

The self-energy is, in $n$ dimensions and up to factors of the coupling constant,

$$
\begin{aligned}
\Sigma\left(k^{2}, \tilde{k}^{2}\right) & =i \int \frac{d^{n} \ell}{(2 \pi)^{n}} \frac{\cos (\ell \cdot \tilde{k})+1}{\left(\ell^{2}-m^{2}+i \epsilon\right)\left((\ell+k)^{2}-m^{2}+i \epsilon\right)} \\
& =i \int_{0}^{1} d x \int \frac{d^{n} \ell}{(2 \pi)^{n}} \frac{\cos (\ell \cdot \tilde{k})+1}{\left[\ell^{2}+k^{2} x(1-x)-m^{2}+i \epsilon\right]^{2}}
\end{aligned}
$$

where $k$ and $\ell$ are the external and loop momentum respectively. For $\theta_{0 i}=0$, the cosine function does not contain the $\ell_{0}$ component. There is thus no new obstacle compared to ordinary theory in continuing $\ell_{0}$ analytically to its imaginary axis. The analytic properties in $k^{2}$ will still be governed by the denominator while the numerator serves to modulate the weight in each direction according to the spatial NC. Using the subscript $E$ to indicate the Euclidean loop momentum, we have,

$$
\begin{aligned}
\Sigma\left(k^{2}, \tilde{k}^{2}\right) & =-\int_{0}^{1} d x \int \frac{d^{n} \ell_{E}}{(2 \pi)^{n}} \frac{\cos \left(\ell_{E} \cdot \tilde{k}\right)+1}{\left[\ell_{E}^{2}+m^{2}-k^{2} x(1-x)-i \epsilon\right]^{2}} \\
& =\frac{d}{d m^{2}} \int_{0}^{1} d z \int \frac{d^{n} \ell_{E}}{(2 \pi)^{n}} \frac{\cos \left(\ell_{E} \cdot \tilde{k}\right)+1}{\ell_{E}^{2}+m^{2}-k^{2}\left(1-z^{2}\right) / 4-i \epsilon}
\end{aligned}
$$

where we have changed the variable $x \rightarrow z=1-2 x$ and noted that the integrand is even in $z$. An imaginary part develops when the denominator can vanish in the integrated domain, which is possible only when $k^{2} \geq 4 m^{2}$,

$$
\begin{aligned}
\operatorname{Im} \Sigma\left(k^{2}, \tilde{k}^{2}\right) & =\pi \frac{d}{d m^{2}} \int_{0}^{1} d z \int \frac{d^{n} \ell_{E}}{(2 \pi)^{n}}\left[\cos \left(\ell_{E} \cdot \tilde{k}\right)+1\right] \delta\left[\ell_{E}^{2}+m^{2}-k^{2}\left(1-z^{2}\right) / 4\right] \\
& =\frac{4 \pi}{k^{2}} \frac{d}{d m^{2}} \int \frac{d^{n} \ell_{E}}{(2 \pi)^{n}}\left[\cos \left(\ell_{E} \cdot \tilde{k}\right)+1\right] \int_{0}^{1} d z \delta\left(z^{2}-v^{2}\right) \\
& =\frac{2 \pi}{k^{2}} \frac{d}{d m^{2}} \int \frac{d^{n} \ell_{E}}{(2 \pi)^{n}}\left[\cos \left(\ell_{E} \cdot \tilde{k}\right)+1\right] v^{-1} \tau\left(k^{2}-4\left(\ell_{E}^{2}+m^{2}\right)\right)
\end{aligned}
$$

with $v=\sqrt{1-4\left(\ell_{E}^{2}+m^{2}\right) / k^{2}}$. 
On the other hand, the once-subtracted self-energy is

$$
\begin{aligned}
& \Sigma\left(k^{2}, \tilde{k}^{2}\right)-\Sigma\left(\mu^{2}, \tilde{k}^{2}\right)=\left(k^{2}-\mu^{2}\right) \frac{d}{d m^{2}} \int_{0}^{1} d z \int \frac{d^{n} \ell_{E}}{(2 \pi)^{n}} \\
& \times \frac{\left[\cos \left(\ell_{E} \cdot \tilde{k}\right)+1\right]\left(1-z^{2}\right) / 4}{\left[\ell_{E}^{2}+m^{2}-k^{2}\left(1-z^{2}\right) / 4-i \epsilon\right]\left[\ell_{E}^{2}+m^{2}-\mu^{2}\left(1-z^{2}\right) / 4-i \epsilon\right]} .
\end{aligned}
$$

Changing the variable $z \rightarrow k^{\prime 2}=4\left(\ell_{E}^{2}+m^{2}\right) /\left(1-z^{2}\right)$ gives,

$$
\begin{aligned}
\Sigma\left(k^{2}, \tilde{k}^{2}\right)-\Sigma\left(\mu^{2}, \tilde{k}^{2}\right) & =\left(k^{2}-\mu^{2}\right) \frac{d}{d m^{2}} \int \frac{d^{n} \ell_{E}}{(2 \pi)^{n}}\left[\cos \left(\ell_{E} \cdot \tilde{k}\right)+1\right] \\
& \times \int_{4\left(\ell_{E}^{2}+m^{2}\right)}^{\infty} \frac{d k^{\prime 2}}{k^{\prime 2}} \frac{2 v^{\prime-1}}{\left(k^{\prime 2}-k^{2}-i \epsilon\right)\left(k^{\prime 2}-\mu^{2}-i \epsilon\right)} \\
& =\left(k^{2}-\mu^{2}\right) \frac{d}{d m^{2}} \int_{4 m^{2}}^{\infty} \frac{d k^{\prime 2}}{k^{\prime 2}} \int \frac{d^{n} \ell_{E}}{(2 \pi)^{n}}\left[\cos \left(\ell_{E} \cdot \tilde{k}\right)+1\right] \\
& \times v^{\prime-1} \tau\left(k^{\prime 2}-4\left(\ell_{E}^{2}+m^{2}\right)\right) \frac{2}{\left(k^{\prime 2}-k^{2}-i \epsilon\right)\left(k^{\prime 2}-\mu^{2}-i \epsilon\right)},
\end{aligned}
$$

with $v^{\prime}=\sqrt{1-4\left(\ell_{E}^{2}+m^{2}\right) / k^{\prime 2}}$. The above equation will be in the desired form if we can pass the operation $d / d m^{2}$ through the integral over $k^{\prime 2}$. We show below that this is indeed permitted since the resulting additional term corresponding to evaluating the integrand at $k^{\prime 2}=4 m^{2}$ actually vanishes. To see this, consider the relevant radial factor of its $\ell_{E}$ integral at $k^{2}=4 m^{2}(1+\eta)$ in the limit of $\eta \rightarrow 0^{+}$,

$$
\begin{aligned}
\text { integral } & \sim \int d \ell_{E} \ell_{E}^{n-1} v^{\prime-1} \tau\left(k^{\prime 2}-4\left(\ell_{E}^{2}+m^{2}\right)\right) \\
& \sim \int_{0}^{\eta} d y y^{(n-2) / 2}(\eta-y)^{-1 / 2} \\
& \propto \eta^{(n-1) / 2}
\end{aligned}
$$

which indeed vanishes as $\eta \rightarrow 0^{+}$. We have ignored the cosine factor which is smooth at $\ell_{E} \sim 0$. Actually, the vanishing of the additional term is just a reflection of the fact that $\operatorname{Im} \Sigma$ vanishes at the physical threshold due to kinematical factors. The dispersion relation eq. (14) is thus established at one loop in perturbation theory. We also notice that, since the subtraction is made with respect to $k^{2}$ but for a fixed value of $\tilde{k}^{2}$, the $\mathrm{UV} / \mathrm{IR}$ mixing is automatically removed from the relation.

\section{Dispersion relation for forward scattering}

The aim of dispersion relations is to provide useful relations amongst measurable quantities on some basic assumptions and independently of perturbation theory in particular. It is already a difficult task to derive a dispersion relation for a general two-by-two scattering amplitude in axiomatic field theory on ordinary spacetime. While microcausality is a 
necessary requirement for this, it is usually not sufficient to guarantee simple dispersion relations. The amplitude depends on three Mandelstam variables which are constrained for a physical process. The analytic properties in one variable, which are the central part of dispersion relations, are inevitably entangled with those in other variables, making things involved. When one tries to represent dispersion integrals in terms of measurable quantities, one is usually afflicted with contributions from unphysical domains. In NC field theory the situation becomes more difficult and unclear. Even if we insist on other basic assumptions, we only have part of spacetime symmetries for special cases of $\theta_{\mu \nu}$, making the corresponding arguments less powerful. Related to this is the appearance of new variables involving NC momenta on which the amplitude can depend. These variables are not completely free parameters but are more or less constrained by ordinary variables for a physical process. This will cause new difficulties. Nevertheless, we would like to make a first attempt on this topic and show where the new difficulties may arise.

We consider the simplest case of forward scattering of a massless scalar particle against a massive one. This is the analogue of the forward photon-nucleon scattering in ordinary theory which has no unphysical regions in dispersion integrals. We assume that the massive particle is at rest in the reference frame where $\theta_{\mu \nu}$ is assigned its value. Note that this is a nontrivial assumption due to the loss of spacetime symmetries; we are only left with boosts in the direction perpendicular to the NC plane and rotations in the plane. And it is the simplest possibility in the sense that we only have one ordinary variable and one NC variable. Following the usual manipulation, the scattering amplitude is,

$$
\mathcal{A}=i \int d^{4} x e^{i k \cdot x} \tau\left(x_{0}\right)\langle M|[j(x), j(0)]| M\rangle,
$$

up to terms which do not affect extraction of analytic properties in energy. $k^{\mu}=\omega\left(1, \vec{e}_{k}\right)$ with $\vec{e}_{k} \cdot \vec{e}_{k}=1$ is the four-momentum of the massless scalar with source $j$, and $M$ is the mass of the massive scalar. Inserting the completeness relation of the physical spectrum and using translational invariance, we have,

$$
\begin{aligned}
\langle M|j(x) j(0)| M\rangle & =\sum_{\alpha} \int \frac{d^{3} \vec{p}}{(2 \pi)^{3} 2 E_{\vec{p}, \alpha}}\langle M|j(x)| \vec{p}, \alpha\rangle\langle\vec{p}, \alpha|j(0)| M\rangle \\
& =\sum_{\alpha} \int \frac{d^{3} \vec{p}}{(2 \pi)^{3} 2 E_{\vec{p}, \alpha}}|\langle M|j(0)| \vec{p}, \alpha\rangle|^{2} e^{i M x_{0}} e^{-i p_{+} \cdot x} .
\end{aligned}
$$

Denoting as $f_{\alpha}\left(\tilde{p}^{2}\right)$ the matrix element squared which depends on $p$ only in the form of $\tilde{p}^{2}$ according to our previous discussion, we obtain,

$$
\langle M|j(x) j(0)| M\rangle=\sum_{\alpha} e^{i M x_{0}} f_{\alpha}\left((i \tilde{\partial})^{2}\right) D\left(x, m_{\alpha}^{2}\right)
$$


For $\theta_{0 i}=0$, the derivatives do not involve $x_{0}$ so that

$$
\mathcal{A}=i \int d^{4} x e^{i k \cdot x} \tau\left(x_{0}\right) \sum_{\alpha} f_{\alpha}\left((i \tilde{\partial})^{2}\right)\left[e^{i M x_{0}} D\left(x, m_{\alpha}^{2}\right)-e^{-i M x_{0}} D\left(-x, m_{\alpha}^{2}\right)\right]
$$

Denoting the sum over $\alpha$ as $i^{-1} f\left(x_{0}, x_{\|}^{2}, \vec{x}_{\perp}^{2}\right)$ which can depend on the relevant variables in the indicated way, we arrive at,

$$
\mathcal{A}=\int d^{4} x e^{i \omega\left(x_{0}-\vec{e}_{k} \cdot \vec{x}\right)} \tau\left(x_{0}\right) f\left(x_{0}, x_{\|}^{2}, \vec{x}_{\perp}^{2}\right)
$$

To proceed further, we have to impose some microcausality requirement to provide support properties in eq. (25) so that analytic continuation in energy may become possible. Since Lorentz invariance is generally lost on $\mathrm{NC}$ space with $\theta_{0 i}=0$, it seems to make no sense to speak of space-like or time-like intervals when a nonzero interval occurs in the NC plane. As we described in the introduction, however, Lorentz invariance still remains in the normal direction to the NC plane. We thus propose the following microcausality requirement to replace the usual one,

$$
[j(x), j(0)]=0, \text { for } x_{0}^{2}-x_{3}^{2}<0,
$$

where we have assumed without loss of generality that NC is restricted to the (12)-plane. This is a weaker requirement than the usual one in the sense that a smaller region in spacetime is excluded as unphysical: while $x_{0}^{2}-x_{3}^{2}<0$ implies $x^{2}<0$, it makes no restriction on $x_{1,2}$.

To show the viability of the above assumption, we compute the following commutator which arises at first order in perturbative $\varphi^{3}$ theory [10],

$$
G=[(\varphi \star \varphi)(x),(\varphi \star \varphi)(y)]
$$

where $\varphi$ is a free field with mass $m$. We have

$$
\begin{aligned}
G= & (\varphi(x) \varphi(y)) \star i \Delta\left(z, m^{2}\right)+i \Delta\left(z, m^{2}\right) \star(\varphi(y) \varphi(x)) \\
& +\varphi(x) \star_{x} i \Delta\left(z, m^{2}\right) \star_{y} \varphi(y)+\varphi(y) \star_{y} i \Delta\left(z, m^{2}\right) \star_{x} \varphi(x),
\end{aligned}
$$

where $z=x-y, \star=\star_{x} \star_{y}$ and $\star_{x}(y)$ refers to the star product with respect to $x(y)$. Since $\Delta\left(z, m^{2}\right)=0$ for $z^{2}<0$, it would be tempting to conclude that $G$ also vanishes. But this is actually very subtle due to the presence of the star product which involves derivatives of infinite order. The highly nonlocal character of the star product may very likely prohibit us from setting $z$ a space-like value before the multiplication is finished. It 
is not clear whether it is mathematically possible to circumvent this problem by a proper extension of distributions. Instead, what is clear is that $G$ vanishes for $z_{0}^{2}-z_{3}^{2}<0$ : the star multiplication has nothing to do with $z_{0}$ and $z_{3}$ and is thus commutable with the procedure of setting a value to $z_{0}^{2}-z_{3}^{2}$, while $z_{0}^{2}-z_{3}^{2}<0$ guarantees $z^{2}<0$ and thus the vanishing of $\Delta$ and $G$.

The above statement can be made more transparent. Let us consider the following typical quantity appearing in $G$,

$$
\begin{aligned}
& (g(x) h(y)) \star D\left(z, m^{2}\right) \\
= & (2 \pi)^{-3} \int d^{2} \vec{p}_{\perp}\left(g(x) \star_{x} e^{+i \vec{p}_{\perp} \cdot \vec{x}_{\perp}}\right)\left(h(y) \star_{y} e^{-i \vec{p}_{\perp} \cdot \vec{y}_{\perp}}\right) \int \frac{d p_{3}}{2 E_{\vec{p}}} e^{-i\left(E_{\vec{p}} z_{0}-p_{3} z_{3}\right)},
\end{aligned}
$$

where $g, h$ can be field operators or c-number functions. For $z_{0}^{2}<z_{3}^{2}$, we make a change of variable, $p_{3}=\gamma\left(p_{3}^{\prime}+\beta E_{\vec{p}^{\prime}}\right)$ with $\beta=z_{0} / z_{3}$ and $\gamma=\left(1-\beta^{2}\right)^{-1 / 2}$, which amounts to a Lorentz boost of the momentum in the 3-direction. Using $d p_{3} / E_{\vec{p}}=d p_{3}^{\prime} / E_{\vec{p}^{\prime}}$ and $E_{\vec{p}} z_{0}-p_{3} z_{3}=-p_{3}^{\prime} z_{3}^{\prime}$, with $z_{3}^{\prime}=\gamma^{-1} z_{3}$ and $z_{0}^{\prime}=0$, and dropping the prime in $\vec{p}$, the above becomes

$$
(2 \pi)^{-3} \int d^{2} \vec{p}_{\perp}\left(g(x) \star_{x} e^{+i \vec{p}_{\perp} \cdot \vec{x}_{\perp}}\right)\left(h(y) \star_{y} e^{-i \vec{p}_{\perp} \cdot \vec{y}_{\perp}}\right) \int \frac{d p_{3}}{2 E_{\vec{p}}} e^{+i p_{3} z_{3}^{\prime}}
$$

For $(g(x) h(y)) \star D\left(-z, m^{2}\right)$, upon $\vec{p} \rightarrow-\vec{p}$, it becomes equal to the above. We thus arrive at,

$$
(g(x) h(y)) \star \Delta\left(z, m^{2}\right)=0, \text { for } z_{0}^{2}-z_{3}^{2}<0 .
$$

This calculation also makes it clear that the commutator $G$ vanishes for any power of fields when $z_{0}^{2}-z_{3}^{2}<0$. This lends perturbative supports to the assumption in eq. (26).

Let us now return back to eq. (25). Due to the weaker microcausality, the integrand can be nonvanishing for $x_{0}>\left|x_{3}\right|\left(x_{3}=x_{\|}\right)$. This is a larger region than the usual forward light cone, where the phase is neither positive- nor negative-definite. The direct analytic continuation to the complex $\omega$ plane is thus not possible. To make the phase positive-definite, we first finish integration over $\vec{x}_{\perp}$ and obtain formally,

$$
\mathcal{A}=\int d x_{0} d x_{3} e^{i\left(\omega x_{0}-k_{3} x_{3}\right)} \tau\left(x_{0}\right) g\left(x_{0}, x_{3}^{2} ; \omega_{\perp}\right)
$$

with $\omega_{\perp}=\left|\vec{k}_{\perp}\right|$. As the integrand is even in $x_{3}$, we may replace $k_{3}$ by its absolute value,

$$
\mathcal{A}\left(\omega ; \omega_{\perp}\right)=\int d x_{0} d x_{3} e^{i\left(\omega x_{0}-\sqrt{\omega^{2}-\omega_{\perp}^{2}} x_{3}\right)} \tau\left(x_{0}\right) g\left(x_{0}, x_{3}^{2} ; \omega_{\perp}\right) .
$$


The problem has been reduced to a $(1+1)$ dimensional one with an effective mass of $\omega_{\perp}$. Let us just press on to see whether it is possible to write down a dispersion relation for $\mathcal{A}$ as we deal with the forward scattering of massive particles in ordinary theory. Since $\operatorname{Im} \sqrt{\omega^{2}-\omega_{\perp}^{2}}>\operatorname{Im} \omega$ for $\operatorname{Im} \omega>0$, continuation to the upper half of the complex $\omega$ plane is still not possible for $\omega_{\perp} \neq 0$. To identify the problem, we write,

$$
\mathcal{A}\left(\omega ; \omega_{\perp}\right)=\int_{0}^{\infty} d r \mathcal{A}\left(r, \omega ; \omega_{\perp}\right)
$$

where

$$
\mathcal{A}\left(r, \omega ; \omega_{\perp}\right)=2 \cos \left(r \sqrt{\omega^{2}-\omega_{\perp}^{2}}\right) \int_{r}^{\infty} d t e^{i \omega t} g\left(t, r^{2} ; \omega_{\perp}\right) .
$$

$\mathcal{A}\left(r, \omega ; \omega_{\perp}\right)$ is analytic in $\omega$ for $\operatorname{Im} \omega>0$ and finite $r$, since the phase factor never blows up and the cosine factor is actually a function of $r^{2}\left(\omega^{2}-\omega_{\perp}^{2}\right)$ which is analytic and does not introduce branch cuts. We can therefore write down a dispersion relation for it,

$$
\mathcal{A}\left(r, \omega ; \omega_{\perp}\right)=\frac{1}{\pi} \int_{-\infty}^{\infty} d \omega^{\prime} \frac{\operatorname{Im} \mathcal{A}\left(r, \omega^{\prime} ; \omega_{\perp}\right)}{\omega^{\prime}-\omega-i \epsilon} .
$$

For $\mathcal{A}\left(r, \omega ; \omega_{\perp}\right)$ decaying not fast enough as $|\omega| \rightarrow \infty$, it is necessary to make subtractions with respect to $\omega$ and for fixed $r$ and $\omega_{\perp}$. To convert the above dispersion relation to the one for $\mathcal{A}\left(\omega ; \omega_{\perp}\right)$ we have to integrate over $r$. This would be done if it were permitted to interchange the order of $r$ and $\omega^{\prime}$ integration. Indeed, for $\left|\omega^{\prime}\right|>\omega_{\perp}$ this is permitted since the cosine factor remains finite in the whole region of $r$. However, this is not the case in the unphysical interval $\left|\omega^{\prime}\right|<\omega_{\perp}$ where the cosine factor blows up as $r \rightarrow \infty$. This is apparently similar to the situation with massive particles in ordinary theory up to an essential difference. Namely, while the problem is reduced to be $(1+1)$ dimensional, the mass shell conditions for physical states are still $(1+3)$ dimensional: the effective mass $\omega_{\perp}$ is not a fixed physical parameter but varies with the scattering configuration. This implies that the above unphysical interval could not be associated with a pole contribution as it is in ordinary theory. This difficulty resulting from a weaker microcausality prevents us from writting a dispersion relation for the scattering amplitude.

Finally, we comment briefly on a special configuration and the case with a massive incident particle. For $\omega_{\perp}=0$ where the forward scattering occurs exclusively in the commutative direction, the above unphysical region disappears so that there is no difficulty to write a dispersion relation for the amplitude. This difference to ordinary theory, i.e., the dependence on the detailed kinematic configurations, can be traced to Lorentz symmetry violation in $\mathrm{NC}$ field theory. When the incident particle has the mass $m$, the above 
unphysical region splits into two subintervals. The contribution from the one with $\left|\omega^{\prime}\right|<$ $m$ can still be attributed to a pole term as in ordinary theory with the modification that its residue depends on $\omega_{\perp}$. The subinterval with $m<\left|\omega^{\prime}\right|<\sqrt{\omega_{\perp}^{2}+m^{2}}$ is afflicted with the same problem discussed above and presents an obstacle to dispersion relations.

\section{Conclusion and discussion}

The phase-like nonlocal nature of NC field theory makes its analytic properties very different from ordinary theory. These properties are intimately related to the issues of unitarity and causality. In this work we tried to incorporate them in the spectral representation and dispersion relations by following the development in axiomatic field theory on ordinary spacetime.

We analyzed spacetime symmetries on NC spacetime that provide a necessary piece of argument in deriving dispersion relations. The NC kinematic variables appear naturally as parametric ones and the analytic properties are studied with respect to the usual kinematic variables. This offers a close similarity to ordinary theory as is illustrated in our perturbative example: as required by unitarity, an imaginary part in amplitude develops only when a physical threshold is crossed. On the other hand, the symmetry analysis forces us to impose a microcausality requirement that is weaker than the usual one; namely, observables commute when they are separated by an interval that is spacelike in the commutative direction. The viability of the assumption is supported by the perturbative calculation of a typical commutator. Starting from this weaker microcausality, however, there arises a new unphysical region when deriving dispersion relations for forward scattering. Since the region is specified by a kinematic variable in the NC plane instead of a mass parameter, it does not seem to be associated to a pole term. It therefore cannot be surmountable using the usual tricks in ordinary theory.

Our treatment of ordinary variables and NC variables hints at a possible subtraction and renormalization scheme that may apply generally. Whenever possible, dispersion relations are written for fixed $\mathrm{NC}$ variables while ordinary variables are integrated over. If there is a UV divergence, subtractions are to be made at the same $\mathrm{NC}$ variables. The planar and nonplanar contributions are thus treated on the same footing as can be seen in our perturbative example. This is in contrast to the popular treatment in the literature that causes the UV/IR mixing problem. We also add in passing that the mixing is not 
necessarily associated with the infrared; if subtraction and renormalization is made with respect to a nonvanishing motion in the $\mathrm{NC}$ plane, the mixing enters when the motion is restricted in the commutative direction. This idea will be further elaborated elsewhere.

\section{References}

[1] S. Minwalla, M. V. Raamsdonk and N. Seiberg, Noncommutative perturbative dynamics, J. High Energy Phys. 02 (2000) 020 hep-th/9912072]; I. Ya. Aref'eva, D. M. Belov and A. S. Koshelev, Two-loop diagrams in noncommutative $\phi_{4}^{4}$ theory, Phys. Lett. B476 (2000) 431 hep-th/9912075; M. V. Raamsdonk and N. Seiberg, Comments on noncommutative perturbative dynamics, J. High Energy Phys. 03 (2000) 035 hep-th/0002186]; A. Matusis, L. Susskind and N. Toumbas, The UV/IR connection in the noncommutative gauge theories, J. High Energy Phys. 12 (2000) 002 hep-th/0002075].

[2] T. Filk, Divergences in a field theory on quantum space, Phys. Lett. B376 (1996) 53; M. Chaichian, A. Demichev and P. Presnajder, Quantum field theory on noncommutative space-times and the persistence of ultraviolet divergences, Nucl. Phys. B567 (2000) 360 hep-th/9812180.

[3] J. Gomis and T. Mehen, Space-time noncommutative field theories and unitarity, Nucl. Phys. B591 (2000) 265 hep-th/0005129.

[4] D. Bahns, S. Doplicher, K. Fredenhagen and G. Piacitelli, On the unitarity problem in space-time noncommutative theories, Phys. Lett. B533 (2002) 178 hep-th/0201222;

see also: C. Rim and J. H. Yee, Unitarity in space-time noncommutative field theories, hep-th/0205193.

[5] Y. Liao and K. Sibold, Time-ordered perturbation theory on noncommutative spacetime: basic rules, hep-th/0205269; Time-ordered perturbation theory on noncommutative spacetime II: unitarity, hep-th/0206011; both to appear in Eur. Phys. J. C.

[6] N. Seiberg, L. Susskind and N. Toumbas, Space-time noncommutativity and causality, J. High Energy Phys. 06 (2000) 044 hep-th/0005015; L. Alvarez-Gaume and J.L.F. Barbon, Nonlinear vacuum phenomena in noncommutative QED, Int. J. Mod. Phys. A16 (2001) 1123 hep-th/0006209. 
[7] M. Lubo, Maximally localized states and causality in noncommutative quantum theories, Phys. Rev. D65 (2002) 066003 hep-th/0106018.

[8] For the development of dispersion relations and original papers, see for example: G. R. Screaton (Ed.), Dispersion relations (Oliver and Boyd, 1961); S. S. Schweber, An introduction to relativistic quantum field theory (Harper \& Row, 1961); J. D. Bjorken and S. D. Drell, Relativistic quantum fields (McGraw-Hill, 1965).

[9] F. T. Brandt, A. Das and J. Frenkel, Dispersion relations for the self-energy in noncommutatie field theories, hep-th/0206058.

[10] For a similar but different calculation of the commutator and its implications, see: M. Chaichian, K. Nishijima and A. Tureanu, Spin statistics and CPT theorems in noncommutative field theory, hep-th/0209008. 